\title{
EVALUACIÓN IN VITRO DE LA ACTIVIDAD ANTILEISHMANIÁSICA DEL EXTRACTO METANÓLICO DE SIETE PLANTAS MEDICINALES
}

\author{
In vitro evaluation of the antileishmanial activity of methanolic extract of seven medicinal \\ plants
}

Juan P Rojas ${ }^{1}$, Sergio G Ronceros ${ }^{1}$, Olga Palacios ${ }^{2}$

${ }^{1}$ Instituto de Investigaciones Clínicas, Facultad de Medicina. ${ }^{2}$ Instituto de Medicina Tropical, Facultad de Medicina. Universidad Nacional Mayor de San Marcos

\section{RESUMEN}

El propósito del presente estudio fue determinar la actividad antileishmaniásica in vitro del extracto metanólico de 7 plantas medicinales, y su efecto sobre la producción de óxido nítrico en macrófagos. Los extractos, en concentración de $250 \mu \mathrm{g} / \mathrm{mL}$, fueron probados contra amastigotes intracelulares. Los macrófagos peritoneales de ratón infectados con amastigotes de Leishmania peruviana fueron coloreados con Giemsa y examinados microscópicamente para determinar la carga parasitaria por 100 macrófagos. El óxido nítrico se determinó indirectamente por su conversión a nitrito. Piper aduncum (matico) redujo la supervivencia de los amastigotes hasta 51,30\% $(p<0,001)$, también se observó efecto reductor con Eucalyptus globulus (eucalipto) y Punica granatum (granado); mientras que Annona cherimola (chirimoya), Annona muricata (guanábana) y Origanum vulgare (orégano) fueron inactivos. La producción de óxido nítrico por los macrófagos fue incrementada significativamente por efecto de Annona cherimola, que produjo la concentración de 13,50 \pm 1,50 $\mu$ M en comparación con 4,90 $\pm 0,10 \mu \mathrm{M}$ del control $(p<0$,oor). También causaron incremento significativo de nitrito Origanum vulgare $(p<0,05)$ y Punica granatum $(p<0,01)$. Se concluye que Piper aduncum, Eucalyptus globulus y Punica granatum exhiben moderado efecto antileishmaniásico in vitro, y que la producción de óxido nítrico es uno de los probables mecanismos de acción para Punica granatum.

Palabras clave: Plantas medicinales, extracto metanólico, Leishmania, amastigotes, óxido nítrico, Piper aduncum, Eucalyptus globulus, Punica granatum.

\section{SUMMARY}

The purpose of this study was to determine the in vitro antileishmanial activity of the methanol extract of seven medicinal plants, and the effect on nitric oxide production in macrophages. The extracts in concentration of $250 \mathrm{gg} / \mathrm{mL}$ were tested against intracellular amastigotes. Mouse peritoneal macrophages infected with Leishmania peruviana were stained with Giemsa and examined microscopically to determine the parasite load by 100 macrophages. Nitric oxide was determined indirectly by its conversion to nitrite. Piper aduncum (matico) reduced the survival of amastigotes to 51,30\% ( $p<$ o,oor), also observed a slight effect of Eucalyptus globulus (eucalyptus) and Punica granatum (pomegranate), while Annona cherimola (custard), Annona muricata (graviola) and Origanum vulgare (oregano) were inactive. Nitric oxide production by macrophages was increased significantly by the effect of Annona cherimola, which led to concentration of 13,50 $\pm 1,50 \mu \mathrm{M}$ compared with 4,90 $\pm 0,10 \mu \mathrm{M}$ in the control $(p<0,001)$. It also produced significant increase of nitrite with Origanum vulgare $(p<0,05)$ and Punica granatum $(p<0,01)$. Was conclude that Piper aduncum, Eucalyptus globulus and Punica granatum showed moderate antileishmanial effect in vitro, and the production of nitric oxide is one of the possible mechanisms of action for Punica granatum.

Keywords: Medicinal plants, methanol extract, leishmaniasis, amastigotes, nitric oxide, Piper aduncum, Eucalyptus globulus, Punica granatum.

\section{INTRODUCCIÓN}

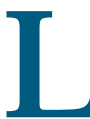

as leishmaniasis presentan un conjunto de síndromes clínicos muy diferentes entre sí, con un espectro que clásicamente ha sido dividido en las formas visceral, cutánea y mucosa, lo cual resulta de la replicación del parásito intracelular obligado, en el sistema fagocítico mononuclear, la dermisy la mucosa naso-orofaríngea, respectivamente. Esta enfermedad es causada por cerca de 21 especies de un protozoario perteneciente al género Leishmania, que son transmitidas porvectores del género Phlebotomus en el Viejo Mundoy Lutzomyia en el Nuevo Mundo ${ }^{(1)}$. 
La leishmaniasis humana amenaza cerca de 350 millones de personas en 98 países o territorios alrededor del mundo y se cree que actualmente están infectadas 12 millones de personas, con un estimado de cerca de 1-2 millones de nuevos casos cada año. La forma cutánea es la más común, con un estimado de 1,5 millones de casos nuevos por año; mientras que la leishmaniasis visceral, que es la forma más severa, llegando a ser fatal si no es tratada, causa 500 ooo casos cada año con más del 90\% de éstos en la India, Bangladesh, Brasil, Nepal y Sudán ${ }^{(2)}$.

En el Perú se han identificado 5 especies de Leishmania: L. (v.) braziliensis, L. (v.) guyanensis, $L$. (v.) peruviana, L. (v.) lainsoni y L. (v.) amazonensis; las cuales están asociadas clínicamente con úlceras cutáneas para L. (v.) guyanensis, L. (v.) peruviana y L. (v.) lainsoni; lesiones cutáneas, cutánea difusa y mucosas para $L$. (v.) amazonensis; y enfermedad mixta cutánea y mucosa para el caso de $L$. (v.) braziliensis. Además, se han considerado como vectores de estos parásitos a pequeñas moscas antropofílicas llamadas en algunas regiones "titira" o "manta blanca", las cuales han sido clasificadas como: Lutzomyia peruensis, L. ayacuchensis, L. verrucarum, L. noguchii, y L. tejadai, de las cuales se ha logrado aislar Leishmania peruviana ${ }^{(3)}$.

La leishmaniasis sería una enfermedad muy antigua en el Perú, tal como se demuestra por la presencia de huacos antropomorfos en la cerámica mochica que presentan mutilaciones de los labios y de la nariz. Actualmente esta enfermedad, endémica en

Tabla 1. Actividad antileishmaniásica in vitro de extractos vegetales a $250 \mu \mathrm{g} / \mathrm{mL}$ sobre amastigotes intracelulares de Leishmania peruviana.

\begin{tabular}{lcc}
\hline \multicolumn{1}{c}{ Muestra } & Amastigotes/100 macrófagos* & $\begin{array}{c}\text { Supervivencia de } \\
\text { amastigotes (\%) }\end{array}$ \\
\hline Annona cherimola & $914,00 \pm 21,28$ & 96,55 \\
Annona muricata & $863,33 \pm 48,56$ & 91,20 \\
Inga edulis & N.d. & N.d. \\
Piper angustifolium & $485,67 \pm 30,43^{* *}$ & 51,30 \\
Origanum vulgare & $886,67 \pm 11,72$ & 93,66 \\
Eucalyptus globulus & $553,67 \pm 38,00^{* *}$ & 58,49 \\
Punica granatum & $581,00 \pm 33,72^{* *}$ & 61,37 \\
Glucantime & $193,33 \pm 35,11^{* *}$ & 20,42 \\
Medio + DMSO 0,10 \% & $946,67 \pm 17,64$ & 100,00 \\
\hline
\end{tabular}

*Valores expresados como media aritmética \pm Desviación estándar

${ }^{* *} p<0,001$ comparado con medio + DMSO 0,10\% (control)

N.d. $=$ No determinado el país, constituye un problema de salud pública que afecta a 19 de sus 24 regiones.

En el período 2000-2007 se reportaron 77,231 casos, de los cuales el $79 \%$ fueron reportados en las regiones de Cusco, Cajamarca y Huánuco. El 6,34\% correspondió a la forma clínica mucosa, indicándose queen el país no se han reportado casos de leishmaniasis visceral. La tendencia a través de los años se mantuvo, observándose incremento en los dos últimos años a expensas de Madre de Dios y las provincias altas de La Libertad ${ }^{(4)}$.

Las drogas disponibles para tratamiento de la enfermedad comprende los antimoniales pentavalentes desarrollados en 1945, que permanecen como medicamentos de primera elección para leishmaniasis visceral y cutánea en la mayor parte del mundo; sin embargo, son tóxicos, pobremente tolerados y el parásito ha generado resistencia. La anfotericina B y la pentamidina son drogas de segunda línea, pero clínicamente muy tóxicas. Si bien se han desarrollado formulaciones lipídicas de anfotericina B, que son menos nocivas, el alto costo limita su amplio uso en países en vías de desarrollo. La miltefosina es el tratamiento oral efectivo para leishmaniasis, pero esta droga puede no ser necesariamente superior a las terapias parenterales y además ha exhibido potencial teratogénico ${ }^{(5)}$.

Estas limitaciones en cuanto a la quimioterapia disponible contra leishmaniasis, genera la necesidad urgente de buscar nuevas drogas y blancos terapéuticos. Los productos vegetales constituyen una alternativa (6), por lo que basandose en los antecedentes, se propone realizar la presente investigación para determinar el efecto antileishmaniásico in vitro del extracto metanólico de las hojas de Origanum vulgare (orégano), Annona muricata (guanábana), Annona cherimola (chirimoya), Punica granatum (granado), Eucalyptus globulus (eucalipto), Inga edulis (guaba) y Piper aduncum (matico), y determinar el efecto que tiene sobre la producción de óxido nítrico 
por los macrófagos, como probable mecanismo de acción.

\section{MATERIALES Y MÉTODOS}

\section{Preparación de los extractos}

Las plantas estudiadas fueron recolectadas en la ciudad de Trujillo, excepto Piper aduncum (Sin. Piper angustifolium) que fue obtenido del Jardín Botánico de la Facultad de Medicina de San Fernando de la Universidad Nacional Mayor de San Marcos. Las hojas de las plantas fueron desecadas a $40^{\circ} \mathrm{C}$ en estufa con aire circulante y luego pulverizadas en un molino de cuchillas. El material pulverizado fue macerado con metanol $\left(\right.$ Fisher $\left.^{\circledR}\right)$ durante 3 días con agitación intermitente; luego se filtró y el filtrado obtenido fue concentrado a sequedad por evaporación del solvente en un rotavapor Büchi ${ }^{\circledR}$. Las muestras obtenidas fueron mantenidas en refrigeración a $2^{\circ} \mathrm{C}$ hasta que fueron utilizadas en los experimentos.

\section{Efecto de los extractos vegetales sobre los amastigotes intracelulares}

Se utilizó el método de Monzote et al. (7). Los macrófagos fueron obtenidos de la cavidad peritoneal de ratones Balb/c por lavado con RPMI-1640 (Sigma ${ }^{\circledR}$ ), suplementado con suero bovino fetal (FBS) al $10 \%$ $\left(\right.$ Sigma $\left.^{\circledR}\right)$. Aproximadamente $4 \times 10^{6}$ macrófagos/ $\mathrm{mL}$ fueron sembrados en placas de 24 pozos por un período de $2 \mathrm{~h}$ a $37^{\circ} \mathrm{C}$ en un incubador con $\mathrm{CO}_{2}$ al $5 \%$. Las células no adheridas fueron removidas mediante un lavado con el medio y se continuó incubando a la misma temperatura por $24 \mathrm{~h}$. Luego se adicionaron $4 \times 10^{7}$ promastigotes de leishmania/mL en cada pozo y se incubó por $6 \mathrm{~h}$ a $37^{\circ} \mathrm{C}$ con $\mathrm{CO}_{2}$ al $5 \%$. Las placas fueron lavadas con RPMI1640 suplementado con FBS, para remover los parásitos no ingeridos, e incubadas por 24 horas en presencia o ausencia de los extractos y de glucantime $(300 \mu \mathrm{g} / \mathrm{mL})$. Los macrófagos incubados sin drogas fueron usados para control (100\% infectados). Los cultivos de macrófagos infectados fueron lavados con FBS, fijados con metanol $\left(\right.$ Fisher $\left.^{\circledR}\right)$, coloreados con
Giemsa $\left(\right.$ Sigma $\left.^{\circledR}\right)$, y examinados con microscopio óptico a $1000 X$ bajo aceite de inmersión. La actividad antileishmaniásica fue determinada calculando el número de amastigotes por 100 macrófagos.

\section{Efecto de los extractos vegetales sobre la producción de óxido nítrico}

La concentración de óxido nítrico se determinó en el sobrenadante de los cultivos de macrófagos peritoneales de ratón. Se agregaron $100 \mu \mathrm{L}$ de la suspensión de macrófagos (4 X $10^{6}$ células $\left./ \mathrm{mL}\right)$ a cada pozo de una microplaca de 96 pozos. Se incubó a $37^{\circ} \mathrm{C}$ por 2 horas en incubador con $\mathrm{CO}_{2}$ al $5 \%$. Se lavó con el medio RPMI 1640 para eliminar las células no adheridas y se agregaron $100 \mu \mathrm{L}$ de medio conteniendo las diferentes concentraciones de las sustancias. Se continuó incubando a $37^{\circ} \mathrm{C}$ por 18 horas. $\mathrm{El}$ óxido nítrico fue determinado indirectamente con la reacción de Griess, que conduce a la formación de cromóforos magenta caracterizados por color grisáceo, según el método de Miranda, et al. ${ }^{(8)}$. Se colectaron 10o $\mu \mathrm{L}$ de sobrenadante y se adicionó el reactivo de Griess, $50 \mu \mathrm{L}$ de reactivo A y $50 \mu \mathrm{L}$ de reactivo B; después de 30 minutos se procedió a la lectura en un lector de microplacas Dynex Technologies Revelation $4,25^{\circledR}$ a una longitud de onda de $540 \mathrm{~nm}$. Los niveles de óxido nítrico se determinaron indirectamente por su conversión a nitritos, usando una curva de calibración estándar.

\section{Análisis de datos}

Los datos obtenidos fueron expresados como media \pm error estándar y las comparaciones entre los

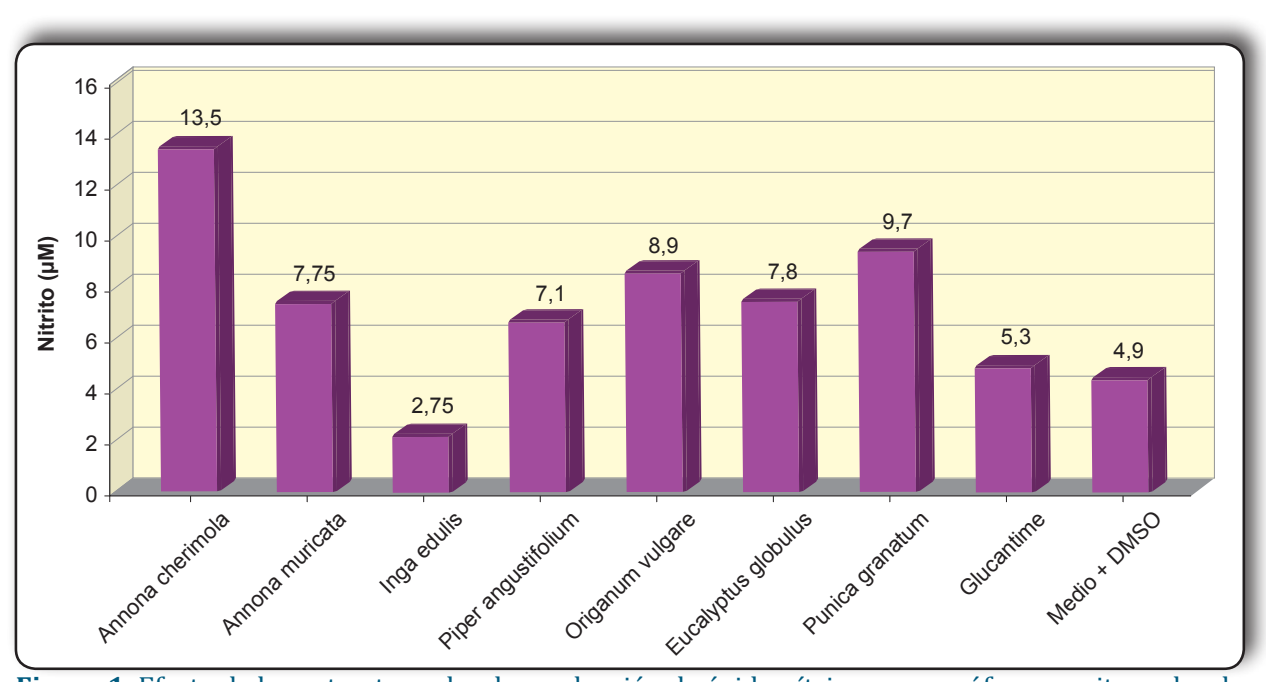

Figura 1. Efecto de los extractos sobre la producción de óxido nítrico en macrófagos peritoneales de ratón 
grupos experimentales fueron realizadas por ANOVA de una vía seguido por la prueba post-hoc de Scheffe. Las medias se consideraron significativamente diferentes cuando $p<0,05$. Se utilizó el software SPSS versión 19,0.

\section{RESULTADOS}

Losextractos metanólicosdelasplantasestudiadas a una concentración de $250 \mu \mathrm{g} / \mathrm{mL}$ mostraron diversa actividad contra amastigotes intracelulares de Leishmania peruviana en macrófagos peritoneales de ratón. Piper aduncum redujo la supervivencia de los amastigotes hasta $51,30 \%(p<o, O 01)$, también se observó un leve efecto de Eucaliptus globulus y Punica granatum; mientras que Annona cherimola, Annona muricata y Origanum vulgare fueron inactivos, como se muestra en la tabla 1.

La producción de óxido nítrico por los macrófagos fue incrementada significativamente por efecto del extracto metanólico de Annona cherimola, que produjo una concentración de 13,50 $\pm 1,50 \mu \mathrm{M}$ en comparación con 4,90 $\pm 0,10 \mu \mathrm{M}$ del control $(p<0, o o r)$. También produjeron incremento significativo de nitrito Origanum vulgare $(p<0,05)$, y Punica granatum $(p<o, o I)$ (figura 1).

\section{DISCUSIÓN}

Debido a que actualmente no se dispone de ninguna vacuna para leishmaniasis y la farmacoterapia de esta enfermedad es insatisfactoria, es importante buscar fuentes alternativas para drogas antiparasitarias. Las especies vegetales producen una gran variedad de metabolitos secundarios con interesantes actividades biológicas: citotóxicas, antiparasitarias y antimicrobianas ${ }^{(9)}$.

Diversos extractos vegetales han mostrado actividad antileishmaniásica en estudios in vitro; los aceites esenciales de algunas plantas, como Allium sativum (ajo) y Rosmarinus officinalis (romero), poseen amplio espectro antimicrobiano, que incluye al parásito Leishmania.

Los metabolitos secundarios de las especies vegetales con actividad antileishmaniásica incluyen flavonoides. Se ha demostrado que dos biflavonas de Podocalyx loranthoides fueron moderadamente activas contra promastigotes de Leishmania mexicana, y se han reportado otros flavonoides, además de alcaloides y triterpenos, con capacidad leishmanicida.

En este estudio se ha demostrado moderada actividad antileishmaniásica in vitro del extracto metanólico de hojas de Piper aduncum, Eucalyptus globulus y Punica granatum cuando se ensayaron contra amastigotes intracelulares en macrófagos peritoneales de ratón (tabla 1). En el ciclo de vida de Leishmania, el amastigote intracelular es el estadio que se da dentro de los mamíferos, y es el blanco para la quimioterapia, se diferencia del promastigote flagelado (forma del parásito en el vector) no sólo en el aspecto morfológico, sino también a nivel bioquímico y molecular: catabolismo de glucosa y utilización de ácidos grasos, nucleasas, proteasas de cisteína, metabolismo de purina, fosforilación de proteínas, expresión génica, y la proteína de superficie de membrana, la metaloproteinasa gp63 y lipofosfoglicano (LPG), lo cual determina una diferente sensibilidad a las drogas, tal como se ha demostrado que los amastigotes son intrínsicamente más de 100 veces sensibles a antimoniales pentavalentes que los promastigotes, con su implicancia en la sensibilidad a las drogas, por lo que no debe cambiarse ${ }^{(10)}$.

El efecto demostrado con Piper aduncum, aunque con una concentración alta $(250 \mu \mathrm{g} / \mathrm{mL})$, guarda relación con el estudio de Torres-Santos, et al. (11), quienes aislaron 2',6'-Dihidroxi-4'-metoxichalcona de las inflorescencias de esta planta con efecto selectivamente tóxico para Leishmania amazonensis, pero hay que tener en cuenta que tanto la procedencia de la planta como la muestra y la especie de leishmania utilizada son diferentes. Las diferentes condiciones del clima, suelo, nutrientes, etc., pueden alterar cualitativa y cuantitativamente la proporción de metabolitos secundarios y por ende el efecto farmacológico. $\mathrm{Si}$ bien las diferentes especies de Leishmania son morfológicamente similares, existen considerables variaciones bioquímicas y moleculares, en particular en los patrones electroforéticos isoenzimáticos y diferencias a nivel de kDNA, los cuales también podría afectar la sensibilidad a las drogas.

Otros estudios reportaron que varias especies de Piper, de diferentes orígenes geográficos, son activos frente a leishmania. En la India, el extracto en éter de petróleo y clorofórmico de la raíz de Piper chaba y el extracto etanólico de las hojas de Piper betle mostraron actividad antileishmania (12). En Brasil, el aceite esencial de Piper claussenianum, el extracto propánico de Piper amalago, y un neolignano aislado de las hojas de Piper regnellii var. pallescens mostraron actividad contra Leishmania amazonensis. También se demostró actividad contra leishmania con el aceite esencial de Piper auritum en Cuba; con Piper elongatum en España; con la fracción hexánica de Piper cumanense 
y Piper holtonii en Colombia; y con una especie no identificada de Piper procedente de Ecuador ${ }^{(13)}$. Estas observaciones resaltan la importancia de las especies del género Piper como una fuente potencial para el aislamiento de drogas leishmanicidas.

La generación de óxido nítrico es una característica de las células del sistema inmune, tales como células dendríticas, células NK, mastocitos y células fagocíticas incluyendo monocitos, macrófagos, microglia, células de Kupffer, eosinófilos y neutrófilos ${ }^{(14)}$. En la leishmaniasis activa, los macrófagos infectados buscan destruir a los parásitos intracelulares a través de elevados niveles de especies reactivas de oxígeno, principalmente $\mathrm{H}_{2} \mathrm{O}_{2}$, y especies reactivas de nitrógeno (óxido nítrico) ${ }^{(15)}$. Es por ello que evaluamos el efecto de los extractos sobre la producción de óxido nítrico en macrófagos peritoneales de ratón, observándose un incremento significativo en el caso de Annona cherimola, Origanum vulgare y Punica granatum (figura 1).

La actividad del extracto metanólico de las hojas de Punica granatum contra amastigotes de Leishmania peruviana, evidenciada en la presente investigación, concuerda con el estudio realizado en Cuba por García et al., quienes reportaron actividad contra Leishmania amazonensis con el extracto hidroalcohólico de la corteza de esta planta ${ }^{(16)}$. Es posible que la reducción de los amastigotes intracelulares por efecto de Punica granatum se deba, en parte, al incremento de la producción de óxido nítrico por los macrófagos. Nunes et al. han demostrado que, la actividad leishmanicida de un alcaloide natural fue dependiente de la activación de la óxido nítrico sintasa inducible (iNOS) que incrementó la síntesis de óxido nítrico por macrófagos, debido probablemente a la unión del alcaloide al sitio catalítico de la iNOS (dominio oxidasa), lo cual activaría la enzima haciendo que los electrones estén disponibles al grupo hem presente en la iNOS, favoreciendo la monooxigenación del sustrato enzimático natural L-arginina, que incrementa la producción de óxido nítrico ${ }^{(17)}$.

La actividad antimicrobiana de la iNOS resultaría de radicales óxido nítrico o S-nitrosotioles o de peroxinitrito (ONOO-). La muerte de parásitos por macrófagos dependiente de la iNOS puede también ser consecuencia de la depleción de arginina, requerida para la síntesis de poliaminas y ADN en Leishmania. Para ciertas cepas de Leishmania se demostró que la L-hidroxiarginina inhibe la actividad de la arginasa en el macrófago y/o parásito y por lo tanto promueve la muerte del parásito ${ }^{(14)}$.
En el caso de Annona muricata, a pesar de haber producido un leve incremento de óxido nítrico, no se observó actividad contra los amastigotes intracelulares; sin embargo, se ha reportado que el extracto metanólico del pericarpio de Annona muricata mostró actividad contra L. (v.) braziliensis, con una concentración efectiva media $>1000 \mu \mathrm{g} / \mathrm{mL}$. Por otra parte, Annona cherimola produjo incremento de óxido nítrico, pero no se observó actividad antileishmania.

Algunos casos producen incremento de óxido nítrico en macrófagos con sustancias de probada actividad antileishmaniásica como el imiquimod (6), en otros casos no se activa la producción de óxido nítrico, sino disminuye como el caso de los triterpenoides: ácido oleanólico y ácido ursólico, que mostraron alta actividad contra amastigotes de Leishmania amazonensis.

Piper aduncum, no produjo incremento significativo de óxido nítrico por los macrófagos, por lo que la actividad contra los amastigotes sería directa sobre el parásito. Sarkar et al. han demostrado efecto leishmanicida directo de Piper betle mediado vía apoptosis, evidenciado por cambios morfológicos, pérdida del potencial de la membrana mitocondrial, fragmentación del ADN y detención del ciclo celular en la subfase $G_{0} / G_{1}{ }^{(12)}$. Torres-Santos et al. también demostraron el efecto inhibitorio directo de Piper aduncum sobre amastigotes de Leishmania amazonensis, mediante estudios ultraestructurales con microscopía electrónica en los que se observó que las mitocondrias fueron agrandadas y desorganizadas. A su vez comprobaron que el efecto leishmanicida no fue debido a la activación del metabolismo oxidativo del nitrógeno en los macrófagos, ya que la producción de óxido nítrico por parte de estos no fue incrementada ${ }^{(11)}$.

\section{CONCLUSIONES}

Bajo estas condiciones experimentales, se concluye que los extractos metanólicos de Piper aduncum (matico), Eucalyptus globulus (eucalipto) y Punica granatum (granado) mostraron moderado efecto antileishmaniásico in vitro, y que la producción de óxido nítrico es uno de los probables mecanismos de acción para Punica granatum.

\section{REFERENCIAS BIBLIOGRÁFICAS}

1. Markle W, Makhoul K. Cutaneous leishmaniasis: recognition and treatment. American Family Physician. 2004; 69(6):1455-146o.

2. World Health Organization. Research priorities for 
chagas disease, human african trypanosomiasis and leishmaniasis. WHO Technical Report Series 975. 2012.

3. Ministerio de Salud. Diagnóstico y tratamiento de la leishmaniosis en el Perú. Norma técnica. Dirección General de Salud de las Personas - MINSA. Lima, 2005.

4. Ministerio de Salud. Norma técnica para el diagnóstico y atención de la leishmaniosis tegumentaria en el Perú. Lima, 2008.

5. Tiuman T, Santos A, Ueda-Nakamura T, Dias B, Nakamura C. Recent advances in leishmaniasis treatment. International Journal of Infectious Diseases 2011; 15: e525-e532.

6. Croft S, Coombs G. Leishmaniasis-current chemotherapy and recent advances in the search for novel drugs. Trends in Parasitology 2003; 19(11): 502-8.

7. Monzote L, Montalvo A, Fonseca L, Pérez R, Suárez M, Rodríguez H. Efecto de derivados de la tiadiazina sobre la capacidad infectiva de promastigotes de Leishmania amazonensis. Rev Cubana Med Trop 2006; 58(1): 25-9.

8. Miranda K, Espey M, Wink D. A rapid, simple spectrophotometric method for simultaneous detection of nitrate and nitrite. Nitric Oxide 2001; 5(1): 62-71.

9. Wink M. Medicinal plants: a source of anti-parasitic secondary metabolites. Molecules 2012; 17(11): 12771-91.

10. Croft S, Yardley V. Chemotherapy of leishmaniasis. Current Pharmaceutical Design 2002; 8(4): 319-42.

11. Torres-Santos E, Lima D, Kaplan M, Meirelles M, RossiBergmann B. Selective effect of 29,69-dihydroxy-49methoxychalcone isolated from Piper aduncum on Leishmania amazonensis. Antimicrobial Agents and Chemotherapy 1999; 43(5):1234-41.

12. Sarkar A, Sen R, Saha P, Ganguly S, Mandal G, Chatterjee M. An ethanolic extract of leaves of Piper betle (Paan)
Linn mediates its antileishmanial activity via apoptosis. Parasitol Res. 2008(6); 102: 1249-55.

13. Gachet MS, Lecaro JS, Kaiser M, Brun R, Navarrete H, Muñoz RA, et al. Assessment of anti-protozoal activity of plants traditionally used in Ecuador in the treatment of leishmaniasis. J Ethnopharmacol 2010; 128(1): 184-97.

14. Bodgan C. Nitric oxide and the immune response. Nature Immunology 2001; 2(10): 907-16.

15. Kolodziej H, Kiderlen A. Antileishmanial activity and immune modulatory effects of tannins and related compounds on leishmania parasited_RAW 264.7 cells. Phytochemistry 2005; 66(17): 2056-71.

16. García M, Monzote L, Scull R, Herrera P. Activity of cuban plants extracts against Leishmania amazonensis. ISRN Pharmacology 2012:1-7.

17. Dos Santos RA, Batista J Jr, Rosa SI, Torquato HF, Bassi CL, Ribeiro TA, et al. Leishmanicidal effect of Spiranthera odoratíssima (Rutaceae) and its isolated alkaloid skimmianine occurs by a nitric oxide dependent mechanism. Parasitology 2011; 138(10): 1224-33.

Manuscrito recibido el: 06/03/2013

Aceptado para su publicación el: 27/03/2013

\section{Correspondencia:}

Nombre: Juan Rojas Armas

Dirección: Instituto de Investigaciones Clínicas

Hospital Nacional Dos de Mayo. Av. Grau cdra 13. Parque Historia de la Medicina. Lima 1, Perú.

Email: jprojasarmas@yahoo.com 